\title{
Influence of Rotational Moulding Process Parameters on Accuracy of Polymer Casts
}

\author{
Tomasz Jachowicz ${ }^{1, *}$, Volodymyr Krasinskyi ${ }^{2}$ \\ 1 Department of Polymer Processing, Mechanical Engineering Faculty, Lublin University of Technology, 36 Nadbystrzycka Street, 20-618 Lublin, Poland \\ 2 Lviv Polytechnic National University, Department of Chemical Technology of Plastics, 79013 Lviv, 12S. Bandera str., Ukraine
}

\begin{abstract}
The present paper describes the experimental tests undertaken to examine the influence of rotational speeds of two axes of the mould on properties of casts produced in the rotational moulding process. The general principles of this polymer processing technology have been described. The main applications have been introduced, and leading advantages and typical disadvantages of rotational moulding process have been told over. Rotational mould's speed around axes was changed, and a thickness of cast walls has been measured also with average surface concentration of colorant in cast walls. Laboratory test stand, processing properties of polymer, also test program and experimental test methodology have been described.
\end{abstract}

\section{Introduction}

The technological process of polymer moulding can be divided into normal and rotational moulding as well as slush moulding and casting. In the rotational moulding process, called shorter as rotomoulding, material in a liquid or plasticized state is placed in a mould's cavity and subjected to centrifugal action or, additionally, to external pressure. In rotomoulding, the mould is rotated around two or more axes at the same time, sometimes with an auxiliary circular motion $[1,2,3]$. In centrifugal casting, which is a type of rotational moulding, the mould is rotated around one axis only. Rotomoulding is predominantly used to produce tanks, containers, cases and other axially symmetric parts, especially those which cannot be produced using other methods of polymer processing due to their exceptionally large external dimensions. Next from the advantages of the rotomoulding technology, in contrast to different methods of production of hollow elements such as blow moulding [4], there is the profitable influence of the processing shrink on removing the cast from the casting mould. Moreover, casting moulds are simpler in the construction and considerably cheaper than injection moulding dies, what also allows to their economic utilization in the low-serial production $[5,6]$. The material applied to rotomoulding has the shape of the powder or micro granules, what it gets the special methods of extruding the most often [7] using special extruder machines with the diverse construction [8]. Moreover, polymers applied to rotomoulding have to be characterize suitable thermal resistance and stability, that not to be subject of the degradation during the decidedly longer time of the processing than near the injection moulding or extrusion $[9,10]$.

The rotomoulding process consists in a correct relation between rotational speeds around the axes, as it fundamentally affects both material flow direction and its even distribution in the mould [1,2]. Adequate technological parameters for the rotomoulding, such as an appropriate ratio of rotational speed around the main axis and an auxiliary one, is fundamental to produce casts with required wall 
thickness. The adequate parameter selection also entails good mixing, which ensures appropriate polymer homogenization in the mould; in effect, the polymer mechanical strength is adequate, and its other properties are satisfactory. Hence, the cast meets the expected functional properties and high quality.

The available literature offers descriptions of studies in the rotomoulding dealing with melt polymer motion in the mould [1], heat exchange between mould and polymer [11, 12], and the behaviour of various polymers in this process [13, 14, 15]. A development of modern calculation methods in recent years has led to developing computer software which allows for the simulation of phenomena occurring during the rotomoulding process [16, 17], mainly phenomena such as polymer heating, flow and cooling, thanks to which the production preparation period is less time consuming and errors can be eliminated at the stage of developing cast design.

\section{Experimental}

To determine the influence of the ratio between main axis speed and auxiliary axis speed of the mould in rotomoulding on wall thickness of produced casts, experimental tests were performed using a laboratory rotomoulding machine available in the Department of Polymer Processing at the Lublin University of Technology. The cast shape resembled a rectangular cube, and some of its edges were rounded. Conducted experimental investigations were connected with the realized investigative programme, as the result of the co-operation with the regional chemical factory which began production of micro granulate designed to fabrication of casts using the rotomoulding process. The factory did not have the possibility of the complex analysis of the foreign literature from the range of the investigations of the rotomoulding, hence undertaken investigations were unique in the national scale, they possessed the cognitive meaning and were used later in industrial conditions during the optimization of the technological parameters of rotomoulding.

The machine control system allows for gearless speed change of both the main and auxiliary axes. The main axis speed can be adjusted within the range from 0 to $33 \mathrm{rpm}$, whereas the auxiliary axis speed is changed within the range from 100 to $0 \%$

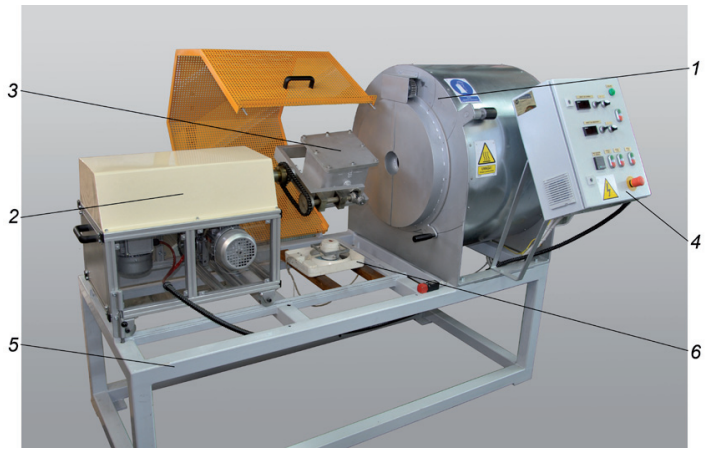

Fig. 1: Laboratory machine for rotational molding: 1 - heating system, 2 - rotational system, 3 - mold, 4 - control and regulation system, 5 - base, 6 - fan.

of the main axis speed. This means that at 100\%, the auxiliary axis makes as many rotations as the main axis, while at 0\% the mould rotation around the auxiliary axis is stopped, as a result of which it is possible to realize the centrifugal moulding process (in which the mould rotates around the main axis only) instead of rotomoulding. Changing the rotational speeds of the main and auxiliary axes is done independently, which therefore makes it possible to adjust the reciprocal ratio of the main axis speed to the auxiliary axis speed [18]. Such possibility is vital for adequate selection of both rotational speeds, which will ensure that casts with adjustable wall thicknesses can be produced. Figure 1 shows this laboratory rotomoulding machine and the mould. The polymer used in the experiment was LUPOLEN 3621 M RM, a medium-density polyethylene PE-MD in powder form, produced by LyondellBasell Industries. This polyethylene kind is used particularly in the rotomoulding due to its good flow rate to density ratio, as well as its good tensile impact strength and thermal stability. The melt flow rate of this polymer is $7.5 \mathrm{~g} / 10 \mathrm{~min}$ (at a temperature of $190^{\circ} \mathrm{C}$ and load of $2.16 \mathrm{~kg}$ ) and its density is $935.5 \mathrm{~kg} / \mathrm{m}^{3}$ [18]. It's Vicat softening temperature is $113^{\circ} \mathrm{C}$, and the recommended processing temperature range is $180 \div 210^{\circ} \mathrm{C}$. To colour the specimens, a colouring concentrate containing a mixture of LLDPE Dowlex 2631 polyethylene and blue colorant Bricofor Blue A4RP was used, wherein the polymer-to-colorant ratio was 1:1. The ratio of the concentrate added to LUPOLEN 3621 M RM, i.e. the polymer the casts were made of, was 1:300.

In the experimental tests, auxiliary axis speed 
was considered a variable factor. Given the capabilities of the control and regulation system of the rotomoulding machine, it was assumed that auxiliary axis speed $n_{\text {sa }}$ was respectively 100, 80 and $60 \%$ of the main axis speed $n_{p a}$. Value of $n_{p a}$ was even 30rpm, so $\mathrm{n}_{\text {sa }}$ had values 30rpm, 24rpm and $18 \mathrm{rpm}$. Ratio of main axis speed $\mathrm{n}_{\mathrm{pa}}$ to auxiliary axis speed nsa, known as coefficient RoR, was calculated with the formula [1]:

$$
R_{o R}=\frac{n_{p a}}{n_{s a}}
$$

Coefficient Ror was equal 1.00; 1.25 and 1.67. Factor examined directly was the thickness of wall $b$ of the produced cast. In order to describe changes in thickness of particular walls of the cast depending on the ratio between main axis speed $n_{p a}$ and auxiliary axis speed $n_{\text {sa }}$, the coefficient of cast wall thickness, Wbs, was determined; it was calculated as the ratio of wall thickness in a given measuring point of the cross section to average wall thickness in a given section.

Investigated in an indirect manner, the mean surface concentration of colorant in particular cast walls was another factor to be examined. In order to determine the mean surface concentration of the colorant, first microscopic images were taken, using selected specimens cut out of the central area of the cast walls, and then the images obtained were analyzed numerically.

The constant factors included such technological parameters of rotational moulding as: main axis speed of $30 \mathrm{rpm}$ and polymer weight of $300 \mathrm{~g}$. The dwell time of the mould in the heating chamber was 20 minutes, while the cooling time of the mould was 25 minutes. The temperature inside the heating chamber was $230^{\circ} \mathrm{C}$ and cooling air flow rate was $300 \mathrm{~m}^{3} / \mathrm{h}$. The disturbing factors included changes in ambient temperature, polymer humidity, air relative humidity and voltage fluctuations in the mains supply. It has been observed that these disturbing factors have no significant effect on the experiment results.

The wall thickness of the produced casts was measured using a slide calliper equipped with a digital measuring device. In order to make the measurements, the casts were cut in three reciprocally perpendicular planes S1, S2 and S3 shown in Figure 2. The measuring points in which the thickness of the sides of the cast was measured were disposed in even spaces, carrying out approximately $10 \mathrm{~mm}$.

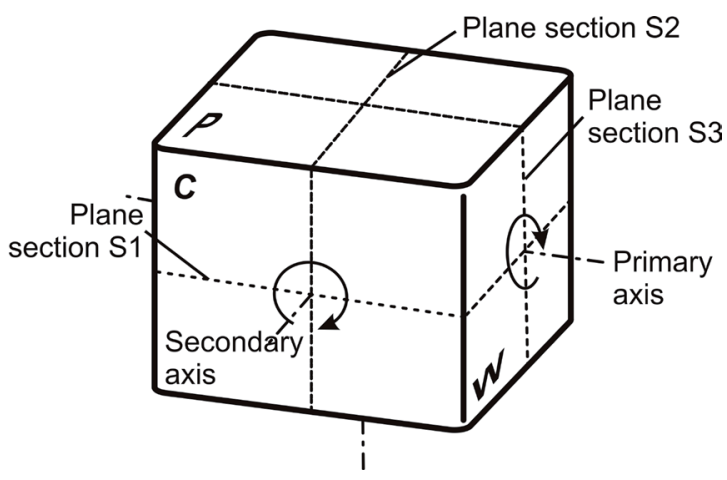

Fig. 2: Positions of plane sections S1, S2 and S3 relative to main and auxiliary axes of rotation.

The microscopic images of the cast wall surfaces were taken using a Vision Engineering DX51 optical microscope, at a magnification of 200 times. The microscopic images could be recorded owing to the use of a Novoflex NDPL-1 (2x) optical adapter that allowed for coupling the microscope with an Olympus E-620 digital camera.

The numerical analysis of the obtained microscopic images was conducted using graphic file editing software such as Paint.NET and XnView. Prior to the analysis of colorant dispersion in the cast walls, the obtained microscopic images had to be verified. In order to take correct readings of the surface areas corresponding to colorant coating, histograms had to be analyzed, as well. Given the conversion of the taken microscopic images to greyscale, the level range was 256 , in shades from white (255) to black (0). To determine the grey level dis-tribution, the histograms of the total area of a microscopic image as well as the areas denoting colorant coating were analyzed. The areas corresponding to colorant coating were marked correctly if the average grey level of the marked areas for the images analyzed had similar values. Average grey level value for the analyzed colorant coating areas had ranges from 23 to 28. After applying the mean grey level of the measured colorant-coated areas of individual microscopic images to a 256-level scale, the mean black saturation with a value of $90 \%$ was obtained.

\section{Experiment results}

Increasing Ror caused a clear change in the melt 
polymer movement and the forming of walls $C$ and W of the cast. At RoR $=1$, in the geometric centre of walls $C$ in the thermal centre resulting from the mass concentration of the journal bearings, average wall thickness was $2.81 \mathrm{~mm}$, and as RoR was increasing, average wall thickness was decreasing. An increase in Ror led to a decrease in cast wall thickness in the rounded corners (in plane section S1). The highest value of wall thickness, over $5 \mathrm{~mm}$, was measured in the acute corners (of plane sections S2 and S3). In the plane perpendicular to the main axis of rotation, the wall thickness on the cast edges changed from $5.32 \mathrm{~mm}$ to $4.60 \mathrm{~mm}$ together with an increase in RoR, whereas in the plane perpendicular to the auxiliary axis of rotation, a change in the value of Rok had no significant effect on changing the wall thickness on the cast edge - it changed from $5.30 \mathrm{~mm}$ to $5.18 \mathrm{~mm}$ together with an increase in RoR.
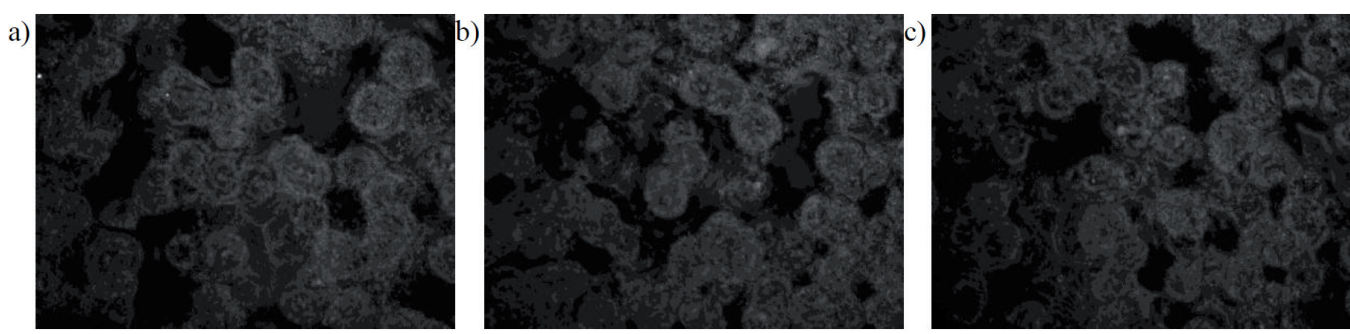

Fig. 4: Microscope image of cast surface showed the colorant surface concentration (magnification 200x): a) wall C RoR=1,00; $\boldsymbol{b}$ ) wall C, RoR=1,25; c) wall C, RoR=1,67.
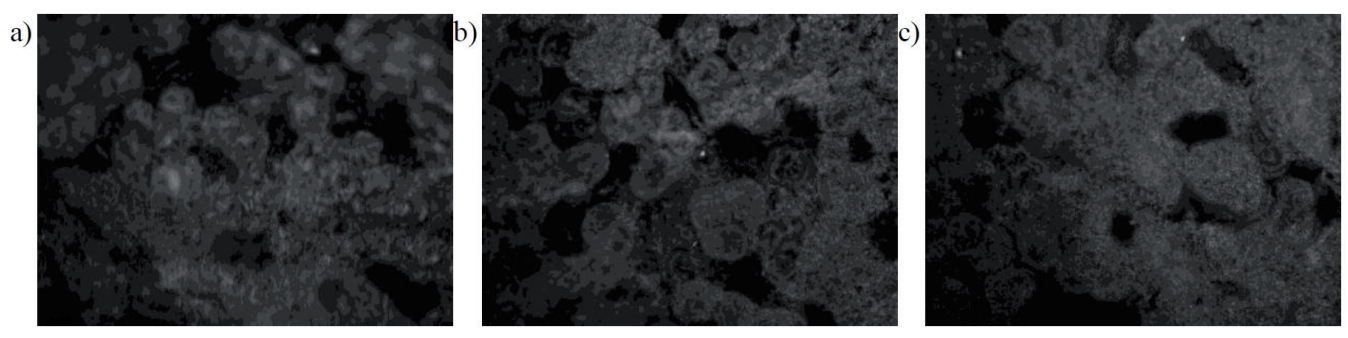

Fig. 5: Microscope image of cast surface showed the colorant surface concentration (magnification 200x): a) wall P RoR=1,00; $\boldsymbol{b}$ ) wall $P$, $R_{0} R=1,25 ;$ c) wall $P, R o R=1,67$.

a)
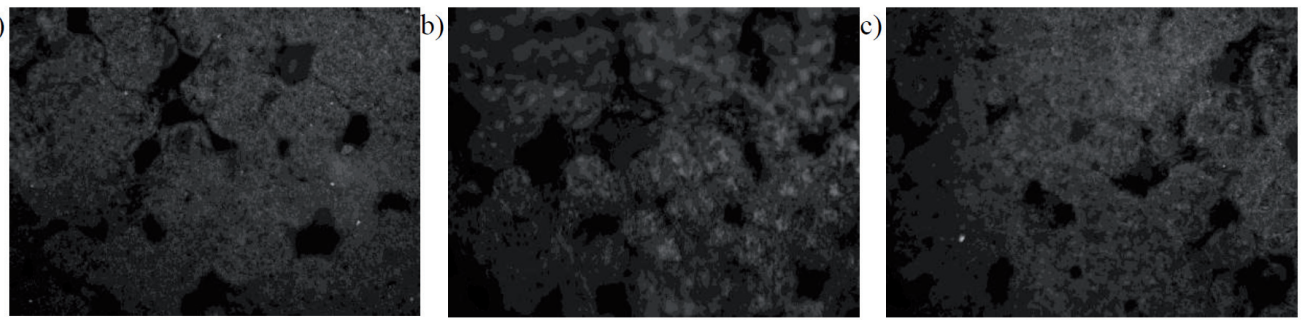

Fig. 6: Microscope image of cast surface showed the colorant surface concentration (magnification 200x): $\boldsymbol{a}$ ) wall W RoR=1,00; $\boldsymbol{b}$ ) wall W, RoR=1,25; c) wall W, RoR=1,67. 
be noted in the case of walls $C$, which resulted from an unfavourable distribution of the mould material mass, that is - the journal bearings connected to the relevant mould walls. An increase in Rok led to a proportional decrease in average wall thickness, the most considerable decrease was observed in the case of walls C (from $2.68 \mathrm{~mm}$ to $2.27 \mathrm{~mm}$, which corresponds to 15\%). In the case of P walls, a decrease in thickness was smaller, by $12 \%$, from $0.92 \mathrm{~mm}$ to $0.81 \mathrm{~mm}$, yet it occurred already at $R_{o R}=1.25$. Average thickness of walls W, determined in their geometric centre (hence on the main axis of rotation) decreased the least, by 10\% (from $0.87 \mathrm{~mm}$ to $0.78 \mathrm{~mm}$ ), and it did not occur until RoR was of 1.67 .

Examples of selected microscopic images obtained from cast walls marked as C, P and W at different values of the RoR coefficient are given in Figure 4, Figure 5 and Figure 6.

\section{Discussion}

In order to describe the extent of changes in the thickness of particular walls depending on the ratio between main axis speeds $n_{p a}$ and auxiliary axis speeds $n_{\text {sa, }}$ wall thickness coefficient Wbs was calculated. In the areas where cast walls were thicker, Wbs was greater than 1, which could especially be observed in the corners and on the edges of the cast, whereas when Wbs was smaller than 1, it meant that the cast walls had a decreased thickness, which was especially noticeable in the central area of the cast walls. Figure 7 illustrates changes in the values of coefficient $W_{\text {bs }}$ depending on the ratio of main axis speed to auxiliary axis speed, determined in plane section S1. The bigger the difference between the mould speed around the main axis and its rotational speed around the auxiliary axis, the more evident the change in cast wall thickness, especially on the walls parallel to the mould axis of rotation. At RoR $=1$, the changes in W bs ranged approximately from 0.60 to 1.3, while at the maximum difference between main axis speed npa and auxiliary axis speed nsa, at RoR = 1.67, differences in the value of $W_{b s}$ ranged from approximately 0.20 up to1.6.

After verifying the selection methodology and marking the areas corresponding to colorant coating, the histogram pairs obtained from individual microscopic images were used to read the value of pixels taken up by the black areas

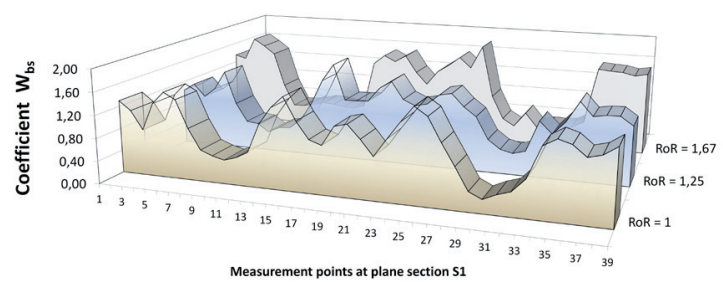

Fig. 7: Changes in values of Wbs depending on speed ratio RoR determined in plane section S1.

of colorant coating. Comparing the number of pixels from the colorant-coated areas with the total number of pixels in a microscopic image, a percentage of the colorant surface concentration was obtained. The values of mean colorant surface concentration obtained for given walls of the casts produced at three different values of the RoR coefficient are presented in Figure 8.

Depending on the changes in Ror coefficient value, the percentage of colorant mean surface concentration had a relatively narrow range: for wall P it ranged from $16.5 \%$ to $20,7 \%$, for wall C from $21.3 \%$ to $25.1 \%$, while for wall W it ranged from $8.9 \%$ to $12.9 \%$. The walls parallel to the main axis of rotation, i.e. P and C, had a similar percentage of the mean colorant surface concentration, being, at the same time, the least sensitive to changing the nonuniformity of rotational speed between the main and auxiliary rotation axes of the casting mould; however, in the case of wall $W$, perpendicular to the main axis of rotation and parallel to the auxiliary axis of rotation, the percentage of mean surface concentration was considerably lower, by about a half. In the case of wall $P$, the initial decrease in the rotational speed of the auxiliary axis, which resulted in increasing the value of the Ror coefficient, did not lead to any significant change in the colorant surface concentration. Such change was not observed until Ror reached its maximum value of 1.67, which caused a decrease in the colorant surface concentration on this wall by approximately $20 \%$. As for wall C, changing the value of the Ror coefficient led to a decrease in the colorant surface concentration; yet no significant dif-ference was observed here when the value of the Ror coefficient was changed from 1.25 to 1.67. The decrease in the colorant surface concentration on wall $\mathrm{C}$ was the lowest and amounted to about $15 \%$. As for wall $W$, the change in the percentage of colorant concentration was similar to the 
one observed for wall C, when changing the RoR coefficient immediately led to reduced colorant amount; nonetheless, wall W exhibited the highest decrease in the percentage of mean colorant surface concentration, amounting to $29 \%$.

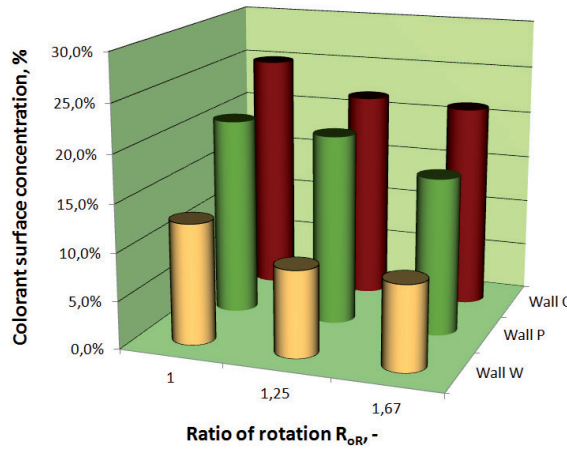

Fig. 8: Dependence of value of coefficient RoR on percentage colorant surface concentration.

The results obtained in the experimental tests undertaken to investigate the influence of changing speed ratio Ror on wall thickness of casts produced by rotational moulding allowed for evaluating the unevenness of wall thickness of the produced casts. An increase in the value of Ror meant a clear difference in wall thicknesses, which resulted in decreased thickness in their geometric centre, while the walls were thicker in the corners and on the edges. Owing to that, the casts in form of rectangular cubes with varying rigidity were produced; the more rigid the casts were, the greater amount of polymer moved towards the cast edges, yet the wall strength was relatively small, which results in worse functional properties of these casts. Decreasing the auxiliary axis speed led to a change in the movement of the plasticized polymer along the walls parallel to this axis, while a relative increase in the main axis speed caused that the melt polymer moved away from walls W1 and W2 which were the least thick. Moreover, it was observed that walls $\mathrm{C} 1$ and C2 were clearly thicker in their geometric centre, which is the area where, in terms of the design, the mould was connected to the journal bearings. This metal mass concentration whose thickness was higher than on walls W1, W2 and P1, P2 leads to the occurrence of a thermal centre that keeps the polymer in the plasticized state in this area longer and leads to uneven cast cooling. As a result, particles of the melt polymer can adhere to this increased temperature area more easily, creating in this way a thicker layer. Compared to the other walls, wall $C$ is nearly twice as thick at RoR $=1$. The unfavourable wall thickness distribution in plane section $C$ was corrected by setting the ratio RoR to a value of 1.25. It should however be noticed that such solution is not optimum - the wall thickness is partially uniform near the journal bearings where the thermal centres occur, yet the thickness of the other walls is decreased because the melt polymer is affected by the centrifugal forces of varying values, which results from a difference between the main axis speed and auxiliary axis speed.

\section{Conclusions}

An increase of the value of coefficient Ror leads to a decrease in average thickness of all cast walls in their geometric centre, which proves that the polymer mass moves towards the corners of the mould form; the largest decrease in average wall thickness has been observed on walls C perpendicular to the auxiliary axis of rotation. The most favourable change in wall thickness has been observed at Ror $=1.25$. Differences of several percent in speeds of both axes entail a clear change in wall thickness which equals tens of percent. In the experiments it was found that decreasing the speed of one axis by $20 \%$ leads to a several percent difference between the thickness of the thickest wall and least thick one in the point wise symmetric cast, which allows for formulating a speculation that it may have a negative effect on strength properties of such cast, for example its rigidity. It follows from this that even small differences in the rotation speed of the axis of rotomoulding machine, caused the mistakes in steering for example, they can cause clear deterioration the exactitude of the fabrication of casts and crossing of the field of the dimension tolerance.

In the case of the mould used to produce casts for the experimental tests, an unfavourable effect of extensive mould material mass concentration has been observed in the area where the journal bearings were located and where the thermal centres occurred. It leads to unevenness of mould cooling and affects negatively wall thickness uniformity.

Received results show on the necessity of the reliable verification of the projects of casting 
moulds not only in the aspect of durability and the correctness of the construction, but also additional thermal analyses connected with absorption, accumulation and emitting warm through the tool.

The clear decrease in the colorant surface concentration in wall $\mathrm{W}$, parallel to the auxiliary axis of rotation and perpendicular to the main axis of rotation of the casting mould, resulted, among others, from the fact that the rotational speed about the auxiliary axis of rotation significantly decreased with increasing the Ror coefficient. As a result, the centrifugal force pressing the plasticized polymer against wall W decreased, while, at the same time, the centrifugal force related to the rotation about the main axis maintained constant value, which caused polymer outflow from wall W onto walls C and $\mathrm{P}$ as well as into the mold corners.

\section{References}

[1] Aissa A. A., Duchesne C., Rodrigue D.: Characterization of polymer powder motion in a spherical mold in biaxial rotation. Polymer Engineering \& Science 2012, 52, 953-963

[2] Crawford R. J, Kearns M. P.: Practical Guide to Rotational Moulding. Second Edition. Smithers Rapra Technology, Shawbury, Shrewsbury, Shropshire, UK 2012.

[3] Jachowicz T:: Technologiczne aspekty odlewania rotacyjnego. Przetwórstwo Tworzyw 2011, 3, 141, 151-155.

[4] Sikora R., Jachowicz T.: Wpływ czasu ochładzania na skurcz przetwórczy wytworów otrzymanych metodą wytłaczania z rozdmuchiwaniem. Polimery 2000, 45, 713-719.

[5] Speich M., Börret R., DeSilva A. K. M., Harrison D. K., Rimkus W.: Precision Mold Manufacturing for Polymer Optics. Materials and Manufacturing Processes 2013, 28, 5, 529-533.

[6] Jeng Yi-Ren, Liu De-Shin, Yau Hong-Tzong: Fast Numerical Algorithm for Optimization Mold Shape of Direct Injection Molding Process. Materials and Manufacturing Processes 2013, 28, 6, 689-694

[7] Sikora J. W.: Chapter 5. Screw extrusion in polymer processing. In: Sabu T., Yang W.: Advances in Polymer Processing: From macro to nano scales. Oxford - Cambrigde - New Delhi, Woodhead Publishing Limited, 2009.

[8] Sikora R., Sasimowski E., Sikora J.W.: Dihelicoidal extrusion. Principles and processing. Polimery 2011, 56, 591-596.

[9] Greskovic F., Dulebova L., Spisak E., Duleba B.: Adhesive wear of selected tool steels used for injection moulds manufacturing. Tribologia: Teoria i praktyka 2013, 44, 1 (247), 47-58.

[10] Bellehumeur C. T., Medina A., Xu H.: Dimensional stability of single-site ethylene co-polymers in rotational molding. Polymer Engineering \& Science 2009, 49, 7, 1400-1409.
[11] Sobotka V., Perot E., Maazouz A., Delaunay D.: Experimental analysis of heat transfer in rotational molding process. International Polymer Processing 2008, 23, 93-102.

[12] Shih-Jung L., Kwang-Hwa F.: Experimental investigation and numerical simulation of the heating/cooling process in rotational molding enhanced with fins. Journal of Applied Polymer Science 2008, 108, 1696-1705.

[13] Kulikov O., Hornung K., Wagner M.: Novel processing additives for rotational molding of polyethylene. International Polymer Processing 2009, 24, 452-462.

[14] Sarrabi S., Colin X., Tcharkhtchi A.: Kinetic modeling of polypropylene thermal oxidation during its processing by rotational molding. Journal of Applied Polymer Science 2010, $118,980-996$.

[15] Ortega Z, Monzón M. D., Benítez A. N., Kearns M., McCourt M., Hornsby R. R.: Banana and Abaca Fiber-Reinforced Plastic Composites Obtained by Rotational Molding Process. Materials and Manufacturing Processes 2013, 28, 8, 879-883.

[16] Wang F., Yao W. G., Qiao C. D., Jia Y. X.: Finite element analysis of the physical gelation process of PVC plastisol during rotational molding. Acta Polymerica Sinica 2012, 9, 1035-1041.

[17] Lim K. K., lanakiev A.: Modeling of rotational molding process: Multi-layer slip-flow model, phase-change, and warpage. Polymer Engineering \& Science 2006, 46, 960-969.

[18] Information taken from the catalogues of Metalchem Toruń and LyondellBasell Industries.

\section{Biographical notes}

Tomasz Jachowicz, Eng. Ph.D.: graduated from the Mechanical Engineering Faculty at Lublin University of Technology in Lublin, located in Poland. He received his doctorate in mechanics and mechanical engineering. He is an assistant professor at the Department of Polymer Processing at the Lublin University of Technology. Fields of research - polymer processing, polymer processing machines and numerical modeling of polymer processing. At present day he is deputy dean for student affairs.

Volodymyr Krasinskyi, Eng. Ph.D.: graduated from the Institute of Chemistry and Chemical Technologies at Lviv Polytechnic National University in Lviv, located in Ukraine (2005). He received his doctorate in Technology of polymer and composite materials (2009). He is an assistant professor at the Department of Chemical Technology of Plastics Processing at the Lviv Polytechnic National University. Fields of research - polymer processing, polymer processing machines, nanocomposites on the basis of thermoplastics, heat-resistant enamels and adhesives. The number of articles in international databases - 16; the number of articles in the national database - 30; number ORCID: http://orcid. org/0000-0001-6374-9340; Scopus Id: http://www.scopus.com/authid/detail. uri?authorld $=37761652800$ 